\title{
To screen or not to screen for obstructive sleep apnea, that is the question
}

The three papers in this journal looking at screening questionnaires for obstructive sleep apnea (OSA) presuppose that screening is a useful and cost-effective activity. However, there is surprisingly little evidence that such activities are valuable, or that they should divert resources away from patients with symptomatic OSA. Screening for OSA by questionnaires, followed by sleep studies, do meet some of the established criteria for introducing screening tests (1). First, OSA is highly prevalent with recent studies suggesting that a degree of OSA can be identified in $25-50 \%$ of adults (2). Second, there is a wealth of data from observational studies linking OSA to adverse outcomes, including cardiovascular disease (3), post-operative complications (4), road traffic collisions (5), and adverse outcomes in pregnancy (6). However, whilst continuous positive airways pressure (CPAP) is clearly an effective treatment for the symptoms of OSA (7), the role of CPAP in preventing other outcomes is unproven, and robust evidence for effective treatments is a vital requirement before screening programs can be justified. We will separately consider screening and its implications for different patient populations.

Severe untreated OSA is associated with increased cardiovascular events although no robust cause and effect data exists (3). The meta-analysis in this journal by Chiu et al. (8), reports that the STOP-BANG questionnaire (SBQ) has the highest sensitivity for identifying patients with severe OSA (pooled sensitivity 0.93 and specificity 0.35 ). Senaratna et al. (9) highlight the issue of generalizability of pooled sensitivities. The pooled sensitivities quoted by Chiu et al. for severe OSA comes from all studies of the SBQ and included no studies of populations with increased cardiovascular risk. In the systematic review by Senaratna et al. populations specifically with increased cardiovascular risk were analyzed separately but there were too few studies, using the Berlin questionnaire (BQ), to perform a meta-analysis in this group. The performance of screening questionnaires for identifying OSA in populations with increased cardiovascular risk is not yet fully established, let alone any subpopulation that might benefit from treatment.

The efficacy of CPAP treatment in improving cardiovascular outcomes is controversial, but is of great importance in making decisions about using screening questionnaires for populations with increased cardiovascular risk. Epidemiological studies have shown rates of cardiovascular disease are as low in patients with treated severe OSA as found in control subjects (3); and randomized controlled trials (RCTs) have shown a benefit of CPAP on surrogate markers of cardiovascular disease such as blood pressure and endothelial function (10)(11). However, the first large RCT, powered to look at hard outcomes, the SAVE trial (12), showed no additional benefit of CPAP above standard care in reducing actual cardiovascular events. This argues strongly against screening for OSA in patients with cardiovascular disease, as CPAP does not reduce subsequent cardiovascular events even in those highly compliant with the therapy. This does not necessarily mean that OSA does not contribute to cardiovascular damage; it may simply be that modern prevention therapies already block any pathophysiological mechanisms from OSA. The SAVE trial excluded the most symptomatic patients but, similarly to others (13), they found significant benefit for patients in terms of symptoms and quality of life. Therefore, patients with cardiovascular disease should not be screened for OSA with the sole intention of reducing cardiovascular 
risk; however we should continue focusing on identifying patients with typical symptoms of OSA, most likely to benefit from investigation and treatment.

Similarly to cardiovascular disease, cohort studies have found associations between OSA and type 2 diabetes (14), and small improvements in glycemic control with CPAP in some studies (15). Short-term RCTs of CPAP in a highly controlled environment have also shown small benefits in glycemic control in pre-diabetic OSA patients (16). Results from longerterm RCTs, that are more representative of clinical practice, are conflicting. The large GLYCOSA trial showed a lack of efficacy of CPAP in improving glycemic control type 2 diabetes patients in patients with associated moderate to severe OSA (17), whereas CPAP lead to reductions in $\mathrm{HbA1c}$ levels in another, albeit smaller, RCT (18). Clearly more work is needed to determine the efficacy of CPAP, in improving glycemic control to an important clinical extent, before trials of screening patients with diabetes can be justified, let alone introduced.

Untreated moderate to severe OSA has been linked with increased post-operative complications (4). Chiu et al. report that the SBQ performed the best in identifying moderate to severe OSA patients (pooled sensitivity 0.90, specificity 0.36) (8) and the SBQ is commonly used to identify these patients in pre-operative assessments. Once more Senaratna et al. raise concerns about the performance of screening in this population; looking at the $B Q$, they were unable to perform a meta-analysis in surgical patients as there were insufficient studies to do so. In observational studies, CPAP treatment has also been linked with reduced post-operative complications (19). However, it is not possible to determine causality from such data. There are several potential confounders to this association, including altered anesthetic regimes, altered intubation technique, and increased monitoring, which are all recommended in anesthetic guidelines for OSA management (20). Importantly, evidence from RCTs has not shown a reduction in postoperative complications with CPAP (21)(22). These studies were small, and few postoperative complications occurred, limiting their power. However, these studies provide the best evidence to date that CPAP has no, or limited, efficacy in reducing post-operative complications. Additionally, identifying and treating OSA with CPAP may not be as important as identifying patients with hypoventilation, as a raised $\mathrm{PaCO}_{2}$ has been reported to be the most important determinant of increased operative risk (23). We are of the opinion that the addition of OSA screening questionnaires to pre-operative screening needs further evaluation to determine its potential benefit and cost effectiveness, despite some advocating widespread screening already (24).

OSA is associated with increased road traffic collisions (5), and CPAP has been associated with reduction of this risk (25), in predominantly non-commercial drivers. Thus it is being suggested that screening and treating professional drivers with OSA, who potentially pose a greater risk, in a timely manner may be of great importance (26). Commercial drivers are less well studied and in the meta-analysis by Chiu et al., only two included studies looked specifically at questionnaire identification of OSA in commercial drivers, with sample sizes of 90 (27) and 100 (28). It is difficult to draw conclusions on the performance of OSA screening questionnaires in professional drivers on this basis. A more recent observational study reported results of a mandatory employer-lead OSA screening program of heavy goods vehicle drivers, with compulsory CPAP treatment for those found to have OSA. They found 
an increased risk of road traffic collisions in employees non-adherent to CPAP compared to those who were adherent (26). The authors proposed that this suggested a benefit of screening and compulsory CPAP treatment for OSA. Of note however, was that even those with only low CPAP adherence equally had no increase in their risk of road traffic collisions compared to controls. We argue that the lack of a dose-related effect of CPAP suggests the alternative hypothesis that patients who reject CPAP altogether are likely to be different in other ways and their increased risk of road traffic collisions may be determined by other risk behaviors. Adherence to CPAP in patients with OSA was required to continue working for the company, even in the absence of symptoms. We would argue that it is important to make the distinction between sleepy and non-sleepy OSA patients, as sleepiness/drowsiness is the likely risk factor for road traffic collisions (29). Unless this distinction is made, and if individuals without symptoms are forced to use CPAP, there is the risk that similar policies will be seen as victimizing such individuals and thus reduce the cooperation required to identify drivers truly at risk. Whilst RCTs seem unethical in patients with OSA and sleepiness, it perhaps would be reasonable to randomize drivers with OSA who do not have symptoms to determine the efficacy of CPAP in this group.

OSA is common in pregnancy and has been associated with maternal and perinatal complications (6)(30)(31). Tankratul et al. found that the BQ was the most studied tool, but had poor-to-fair pooled sensitivity $(0.66)$ and specificity $(0.62)$ for detecting OSA. They conclude that sleep questionnaires are inadequate at detecting OSA in pregnancy and that a new screening tool is needed to detect OSA in pregnancy. We would argue that further work and RCTs need to be done to establish the benefit of CPAP treatment in this group prior to embarking on RCTs to assess screening programs.

Chiu et al. looked at the performance of sleep apnea questionnaires specifically in the subset of studies on sleep clinic populations. In sleep clinic populations there is of course a MUCH higher prevalence of OSA than in the general population, which changes the performance of such questionnaires. Use of the SBQ in this context not surprisingly shows a high sensitivity and poor specificity at 0.90 and 0.41 respectively for detecting OSA (AHI>5). There is little additional diagnostic value of a positive screening questionnaire in such high OSA prevalence groups. In clinical practice, predictors of CPAP benefit might be more useful. It also will not identify other important sleep disorders that may be causing symptoms. We argue that in such a setting, in addition to a skilled history and examination, clinicians should simply proceed straight to an appropriate sleep study.

Both Chiu et al. and Tantrakul et al. found high statistical heterogeneity in the performance of questionnaires between the included studies, which is probably due to many differences in the patient populations studied, particularly the actual OSA prevalence. In addition, the studies included used a combination of polysomnography and home sleep studies as their measure of OSA severity, which will have introduced variability in the AHI/RDI. It is important to note that whilst polysomnography is assumed to be the gold-standard for measuring $\mathrm{AHI}$, it has only weak associations with symptoms (32)(33) and shows considerable night-to-night variation (34). Senaratna et al. showed that the performance of the $B Q$ was dependent not only on the mode of sleep study but also on the specific hypopnea definition. These differences in patient populations and in measurements mean that it is difficult to generalize these results. Furthermore, screening tools for OSA are 
assessed on their ability to detect OSA defined by the AHI, but this is very unlikely to be the best or most appropriate predictor of risk. Other factors such as the severity of intermittent hypoxia (35), or catecholamine surges (36), may be more relevant to cardiovascular disease; and sleep fragmentation (37), may be more important to driving risk.

Our experience in the UK is that screening for OSA, with or without symptoms, in a variety of clinical settings is leading to increasing numbers of referrals and increasing pressure on sleep clinics. Whilst clear epidemiological links exist between OSA and potential complications, there is a lack of evidence for improvements in either post-operative outcomes or road traffic collisions with treatment making screening hard to recommend. Whilst it may not be possible to conduct trials in some populations, such as in sleepy drivers in whom RCTs may be unethical, these are needed in others. The SAVE trial provides the strongest evidence yet to refute the hypothesis of improved cardiovascular outcomes in OSA with CPAP treatment. Therefore, whilst the STOP-BANG questionnaire is the best performing tool to identify risk of OSA as quantified by AHI, further work is first needed to determine if this leads to improved patient outcomes, and then to determine if screening is cost effective. Screening questionnaire performance varies in different populations and thus performance is not generalizable. The poor performance of the $\mathrm{BQ}$ in pregnancy highlights this issue. Robust RCTs are required to determine if there are treatment benefits, followed by further RCTs of screening for OSA to see if any therapeutic benefits subsequently translate into improved care for patients. There are many areas in medicine where assumptions about benefits (and the extent of adverse outcomes) from screening and treatments have not been confirmed when subjected to robust RCTs. Without these trials there is a real danger that futile screening will take resources away from the many symptomatic patients still in need of investigation and treatment (38).

1. Wilson J, Junger G. Principles and practice of screening for disease. Public Health Pap. 1968;

2. Heinzer R, Vat S, Marques-Vidal P, Marti-Soler H, Andries D, Tobback N, et al. Prevalence of sleep-disordered breathing in the general population: the HypnoLaus study. Lancet Respir Med. 2015 Apr;3(4):310-8.

3. Marin JM, Carrizo SJ, Vicente E, Agusti AGN. Long-term cardiovascular outcomes in men with obstructive sleep apnoea-hypopnoea with or without treatment with continuous positive airway pressure: an observational study. Lancet. 2005 Mar 19;365(9464):1046-53.

4. Kaw R, Chung F, Pasupuleti V, Mehta J, Gay PC, Hernandez AV. Meta-analysis of the association between obstructive sleep apnoea and postoperative outcome. $\mathrm{Br} \mathrm{J}$ Anaesth. 2012 Dec;109(6):897-906.

5. Tregear S, Reston J, Schoelles K, Phillips B. Obstructive sleep apnea and risk of motor vehicle crash: systematic review and meta-analysis. J Clin Sleep Med JCSM Off Publ Am Acad Sleep Med. 2009 Dec 15;5(6):573-81. 
6. Pamidi S, Pinto LM, Marc I, Benedetti A, Schwartzman K, Kimoff RJ. Maternal sleepdisordered breathing and adverse pregnancy outcomes: a systematic review and metaanalysis. Am J Obstet Gynecol. 2014 Jan;210(1):52.e1-52.e14.

7. McEvoy RD, Thornton AT. Treatment of obstructive sleep apnea syndrome with nasal continuous positive airway pressure. Sleep. 1984;7(4):313-25.

8. Chiu H-Y, Chen P-Y, Chuang L-P, Chen N-H, Tu Y-K, Hsieh Y-J, et al. Diagnostic accuracy of the Berlin questionnaire, STOP-BANG, STOP, and Epworth sleepiness scale in detecting obstructive sleep apnea: A bivariate meta-analysis. Sleep Med Rev. 2016 Nov 5 ;

9. Senaratna C, Perret J, Matheson M, Lodge C, Lowe A, Cassim R, et al. Validity of the Berlin questionnaire in detecting obstructive sleep apnoea: A systematic review and meta-analysis. Sleep Med Rev.

10. Hu X, Fan J, Chen S, Yin Y, Zrenner B. The role of continuous positive airway pressure in blood pressure control for patients with obstructive sleep apnea and hypertension: a meta-analysis of randomized controlled trials. J Clin Hypertens Greenwich Conn. 2015 Mar;17(3):215-22.

11. Schwarz EI, Puhan MA, Schlatzer C, Stradling JR, Kohler M. Effect of CPAP therapy on endothelial function in obstructive sleep apnoea: A systematic review and metaanalysis. Respirol Carlton Vic. 2015 Aug;20(6):889-95.

12. McEvoy RD, Antic NA, Heeley E, Luo Y, Ou Q, Zhang X, et al. CPAP for Prevention of Cardiovascular Events in Obstructive Sleep Apnea. N Engl J Med. 2016 Sep 8;375(10):919-31.

13. Craig SE, Kohler M, Nicoll D, Bratton DJ, Nunn A, Davies R, et al. Continuous positive airway pressure improves sleepiness but not calculated vascular risk in patients with minimally symptomatic obstructive sleep apnoea: the MOSAIC randomised controlled trial. Thorax. 2012 Dec;67(12):1090-6.

14. West SD, Nicoll DJ, Stradling JR. Prevalence of obstructive sleep apnoea in men with type 2 diabetes. Thorax. 2006 Nov;61(11):945-50.

15. Babu AR, Herdegen J, Fogelfeld L, Shott S, Mazzone T. Type 2 diabetes, glycemic control, and continuous positive airway pressure in obstructive sleep apnea. Arch Intern Med. 2005 Feb 28;165(4):447-52.

16. Pamidi S, Wroblewski K, Stepien M, Sharif-Sidi K, Kilkus J, Whitmore H, et al. Eight Hours of Nightly Continuous Positive Airway Pressure Treatment of Obstructive Sleep Apnea Improves Glucose Metabolism in Patients with Prediabetes. A Randomized Controlled Trial. Am J Respir Crit Care Med. 2015 Jul 1;192(1):96-105.

17. Shaw JE, Punjabi NM, Naughton MT, Willes L, Bergenstal RM, Cistulli PA, et al. The Effect of Treatment of Obstructive Sleep Apnea on Glycemic Control in Type 2 Diabetes. Am J Respir Crit Care Med. 2016 Aug 15;194(4):486-92. 
18. Martínez-Cerón E, Barquiel B, Bezos A-M, Casitas R, Galera R, García-Benito C, et al. Effect of Continuous Positive Airway Pressure on Glycemic Control in Patients with Obstructive Sleep Apnea and Type 2 Diabetes. A Randomized Clinical Trial. Am J Respir Crit Care Med. 2016 Aug 15;194(4):476-85.

19. Mutter TC, Chateau D, Moffatt M, Ramsey C, Roos LL, Kryger M. A matched cohort study of postoperative outcomes in obstructive sleep apnea: could preoperative diagnosis and treatment prevent complications? Anesthesiology. 2014 Oct;121(4):70718.

20. American Society of Anesthesiologists Task Force on Perioperative Management of patients with obstructive sleep apnea. Practice guidelines for the perioperative management of patients with obstructive sleep apnea: an updated report by the American Society of Anesthesiologists Task Force on Perioperative Management of patients with obstructive sleep apnea. Anesthesiology. 2014 Feb;120(2):268-86.

21. O'Gorman SM, Gay PC, Morgenthaler TI. Does autotitrating positive airway pressure therapy improve postoperative outcome in patients at risk for obstructive sleep apnea syndrome? A randomized controlled clinical trial. Chest. 2013 Jul;144(1):72-8.

22. Liao P, Luo Q, Elsaid H, Kang W, Shapiro CM, Chung F. Perioperative auto-titrated continuous positive airway pressure treatment in surgical patients with obstructive sleep apnea: a randomized controlled trial. Anesthesiology. 2013 Oct;119(4):837-47.

23. Kaw R, Bhateja P, Paz Y Mar H, Hernandez AV, Ramaswamy A, Deshpande A, et al. Postoperative Complications in Patients With Unrecognized Obesity Hypoventilation Syndrome Undergoing Elective Noncardiac Surgery. Chest. 2016 Jan;149(1):84-91.

24. Chung F, Memtsoudis SG, Ramachandran SK, Nagappa M, Opperer M, Cozowicz C, et al. Society of Anesthesia and Sleep Medicine Guidelines on Preoperative Screening and Assessment of Adult Patients With Obstructive Sleep Apnea. Anesth Analg. 2016 Aug;123(2):452-73.

25. Tregear S, Reston J, Schoelles K, Phillips B. Continuous positive airway pressure reduces risk of motor vehicle crash among drivers with obstructive sleep apnea: systematic review and meta-analysis. Sleep. 2010 Oct;33(10):1373-80.

26. Burks SV, Anderson JE, Bombyk M, Haider R, Ganzhorn D, Jiao X, et al. Nonadherence with Employer-Mandated Sleep Apnea Treatment and Increased Risk of Serious Truck Crashes. Sleep. 2016 May 1;39(5):967-75.

27. Firat $H$, Yuceege $M$, Demir A, Ardic S. Comparison of four established questionnaires to identify highway bus drivers at risk for obstructive sleep apnea in Turkey: Bus drivers and obstructive sleep apnea. Sleep Biol Rhythms. 2012 Jul;10(3):231-6.

28. Popević MB, Milovanović A, Nagorni-Obradović L, Nešić D, Milovanović J, Milovanović APS. Screening commercial drivers for obstructive sleep apnea: translation and validation of Serbian version of Berlin Questionnaire. Qual Life Res Int J Qual Life Asp Treat Care Rehabil. 2016 Feb;25(2):343-9. 
29. Tefft BC. Prevalence of motor vehicle crashes involving drowsy drivers, United States, 1999-2008. Accid Anal Prev. 2012 Mar;45:180-6.

30. Xu T, Feng Y, Peng H, Guo D, Li T. Obstructive sleep apnea and the risk of perinatal outcomes: a meta-analysis of cohort studies. Sci Rep. 2014 Nov 10;4:6982.

31. Luque-Fernandez MA, Bain PA, Gelaye B, Redline S, Williams MA. Sleep-disordered breathing and gestational diabetes mellitus: a meta-analysis of 9,795 participants enrolled in epidemiological observational studies. Diabetes Care. 2013 Oct;36(10):3353-60.

32. Weaver TE, Maislin G, Dinges DF, Bloxham T, George CFP, Greenberg H, et al. Relationship between hours of CPAP use and achieving normal levels of sleepiness and daily functioning. Sleep. 2007 Jun;30(6):711-9.

33. Macey PM, Woo MA, Kumar R, Cross RL, Harper RM. Relationship between obstructive sleep apnea severity and sleep, depression and anxiety symptoms in newly-diagnosed patients. PloS One. 2010 Apr 16;5(4):e10211.

34. Chediak AD, Acevedo-Crespo JC, Seiden DJ, Kim HH, Kiel MH. Nightly variability in the indices of sleep-disordered breathing in men being evaluated for impotence with consecutive night polysomnograms. Sleep. 1996 Sep;19(7):589-92.

35. Baguet J-P, Barone-Rochette G, Tamisier R, Levy P, Pépin J-L. Mechanisms of cardiac dysfunction in obstructive sleep apnea. Nat Rev Cardiol. 2012 Dec;9(12):679-88.

36. Kohler M, Stradling JR. Mechanisms of vascular damage in obstructive sleep apnea. Nat Rev Cardiol. 2010 Dec;7(12):677-85.

37. Vakulin A, D'Rozario A, Kim J-W, Watson B, Cross N, Wang D, et al. Quantitative sleep EEG and polysomnographic predictors of driving simulator performance in obstructive sleep apnea. Clin Neurophysiol Off J Int Fed Clin Neurophysiol. 2016 Feb;127(2):142835.

38. Rejon-Parrilla JC, Garau M, Sussex J. Obstructive Sleep Apnoea Health Economics Report. British Lung Foundation; 2014.

2000 word limit (2032 main body currently). References in Vancouver style in parentheses or superscript with a maximum of six authors listed. Corresponding address and email at top or the bottom of the article. 